\title{
Discrete Space-Time on the Manifestation of Mass
}

\author{
Rickey W. Austin, PhD
}

Senior Research Scientist, River Song Innovations, Inc, Raleigh, NC USA

Doi: 10.19044/esj.2018.v14n3p31 URL:http://dx.doi.org/10.19044/esj.2018.v14n3p31

\begin{abstract}
Schwarzschild's Metric (Schwarzschild 1916) under specific conditions provides a Taylor series first order discrete length when transforming coordinates between observers. Exploring the consequences of the discrete length produces an a priori result of quantized space-time. Deriving base units from the quantization of space-time and applying elementary charge, exact formulations for the observed Schwarzschild's discrete units are obtained. These units are equivalent to Planck's mass, length, time, momentum, force, energy and Planck's constant (NIST CODATA 2014).
\end{abstract}

Keywords: Gravitational Quanta of Energy, Bohr Radius, Planck's constant, Fine Structure Constant, Heisenberg's uncertainty

\section{Introduction}

The Schwarzschild's Metric, the first exact solution to Einstein's field equations of General Relativity is (Schwarzschild 1916)

$$
c^{2} d \tau^{2}=\left(1-\frac{2 G M}{r c^{2}}\right) c^{2} d \tau^{2}-\left(1-\frac{2 G M}{r c^{2}}\right)^{-1} d r^{2}-r^{2}\left(d \theta^{2}+\sin ^{2} \theta d \varphi^{2}\right)
$$

where $\mathrm{c}$ is the speed of light, $\tau$ is proper time, $\mathrm{G}$ is Newton's universal gravitational constant, $\mathrm{r}$ is the radial distance from the mass generating the field and $\mathrm{t}$ is the time coordinate located an infinite distance from the mass. Consider only a radial change in position of an observer. The transformation of coordinate $r$, with coordinates $t, \theta, \varphi$ unchanged, given by the metric is

$$
c^{2} d \tau^{2}=-\left(1-\frac{2 G M}{r c^{2}}\right)^{-1} d r^{2}
$$

In equation 2 set $x=\frac{M}{r}$ and perform a Taylor series expansion about zero for the RHS. Substitute $\frac{M}{r}$ back in for $\mathrm{x}$ and arrive at the series:

$$
1+\frac{G M}{r c^{2}}+\frac{3 G^{2} M^{2}}{2 r^{2} c^{4}}+\mathcal{O}\left(\left(\frac{M}{r}\right)^{3}\right)
$$


A first order approximation of length $L$ by an observer an infinite distance from the mass, transformed to an observer radial distance $r$ from the mass is:

$$
L^{\prime}=\left(1+\frac{G M}{r c^{2}}\right) L(4)
$$

Where $L^{\prime}$ is the observed length at radial distance $r$. When $r=L$, equation 4 reduces to

$$
L^{\prime}=L+\frac{G M}{c^{2}}
$$

From equation $4, \mathrm{~L}^{\prime}$ is observed as equivalent to $\mathrm{L}$ with an additional length. The value of the additional length is only dependent on $M$ and independent of radial distance. Equation 4 allows equation 5 to be written as (when $\mathrm{r}=\mathrm{L}=\frac{G M}{c^{2}}$ ):

$$
L^{\prime}=2 L=\frac{2 G M}{c^{2}}
$$

In other words, a distance of $\frac{G M}{c^{2}}$ an infinite distance from the mass is observed as $\frac{2 G M}{c^{2}}$ locally at the distance $\frac{G M}{c^{2}}$ from the mass. Reversing the transformation, where $\mathrm{L}^{\prime}=\mathrm{r}=\frac{G M}{c^{2}}$, the transformation takes the form:

$$
L=\frac{L^{\prime}}{2}=\frac{G M}{2 c^{2}}
$$

In equation $7, \mathrm{~L}^{\prime}$ is the length $\frac{G M}{c^{2}}$ as observed at the radial distance $\mathrm{L}$ from the mass transformed to an observer infinitely far away. The transformed length is observed as half the length. Recall, this is a first term Taylor series approximation. Note the cancellation of $r$ in equation 4 of the first term approximation only cancels in the first term. If the approximation is obtained to higher terms, the additional approximations are radial dependent. However, in the higher term approximations the first term approximation will always be independent of radial distance when the condition of $\mathrm{r}=\mathrm{L}$ is applied.

This paper's focus is on the length $\frac{G M}{c^{2}}$. From this length, base units are derived. These units are compared to Planck's units and Planck's reduced constant $\hbar$.

\section{Deriving Base Units}

In the Schwarzschild solution, the mass is invariant (a static solution). Thus, the derived unit length is

$$
\hat{l}_{g}=\frac{G M}{c^{2}}(8)
$$

The invariance of $\mathrm{c}, \mathrm{G}$ and $\mathrm{M}$ imply the length is invariant. The unit time is constructed as the time it takes light to transverse one unit length. 


$$
\hat{t}_{g}=\frac{G M}{c^{3}}
$$

Newtonian gravitational force at one unit length between two masses is (Marion 1995)

$$
\overrightarrow{F_{g}}=\frac{G M^{2}}{\hat{l}_{g}^{2}}=\frac{G M^{2}}{\left(\frac{G M}{c^{2}}\right)^{2}}=\frac{c^{4}}{G}
$$

This value is invariant and not dependent on the mass when unit values are constructed as previously set forth. Energy, a scalar value, is calculated from a force applied over a distance. Thus a unit of gravitational energy may be obtained by

$$
\hat{E}_{g}=\overrightarrow{F_{g}} \cdot \hat{l}=\frac{G M^{2}}{\hat{l}_{g}^{2}}(\hat{l})=\frac{c^{4}}{G}\left(\frac{G M}{c^{2}}\right)=M c^{2}
$$

A unit of gravitational energy delivered for one unit of time is

$$
\hat{E}_{g} \hat{t}=\frac{G M^{2}}{c}(12)
$$

The following base units have been derived for a system containing a single non charged stationary point mass.

\begin{tabular}{|c|c|}
\hline Unit & Value \\
\hline Mass & $\widehat{M}$ \\
\hline Length & $\hat{l}_{g}=\frac{G M}{c^{2}}$ \\
\hline Time & $\hat{t}_{g}=\frac{G M}{c^{3}}$ \\
\hline Gravitational Force & $\vec{F}_{g}=\frac{c^{4}}{G}$ \\
\hline Gravitational Energy & $\hat{E}_{g}=M c^{2}$ \\
\hline A Unit Gravitational Energy for a Unit Time & $\hat{E}_{g} \hat{t}=\frac{G M^{2}}{c}$ \\
\hline
\end{tabular}

\section{Table 1}

\section{Elementary Charge at a Quanta of Length}

Consider two elementary charges, each a point charge, separated by one unit length $\hat{l}$ from equation (8). The Coulomb force between the two charges is (Halliday 1997)

$$
\hat{F}_{e}=\frac{e^{2}}{4 \pi \epsilon_{0} \hat{l}_{g}^{2}}
$$

Where e is the elementary charge, $\epsilon_{0}$ is the vacuum permittivity and $\hat{l}$ is the previously derived unit length. The force applied over the distance of one unit length is the Coulomb unit energy.

$$
\hat{E}_{e}=\overrightarrow{F_{e}} \cdot \hat{l}_{g}=\frac{e^{2}}{4 \pi \epsilon_{0} \hat{l}_{g}^{2}}\left(\hat{l}_{g}\right)=\frac{e^{2}}{4 \pi \epsilon_{0} \hat{l}_{g}}
$$

One Coulomb unit of energy for one unit of time is

$$
\hat{E}_{e} \hat{t}=\left(\frac{e^{2}}{4 \pi \epsilon_{0}\left(\frac{G M}{c^{2}}\right)}\right)\left(\frac{G M}{c^{3}}\right)=\frac{e^{2}}{4 \pi \epsilon_{0} c}
$$


Where unit length and unit time have values substituted from equations 8 and 9. The value is invariant with no dependence on unit mass or unit length. Consider the ratio of energies from equation (11) and (14).

$$
1=k \frac{\vec{E}_{e}}{\vec{E}_{g}}=k \frac{\left(\frac{e^{2}}{4 \pi \epsilon_{0} \hat{l}_{g}}\right)}{M c^{2}}=k \frac{e^{2}}{4 \pi \epsilon \hat{l}_{g} M c^{2}}
$$

here the constant of proportionality $\mathrm{k}$ is introduced. It is an unknown value representing the ratio of the energies. Both energies are central force based and dependent upon the unit distance $\hat{l}_{g}$. In equation 16 , length is the varying factor for changing the magnitude of $\vec{E}_{e}$. As such $\mathrm{k}$ is applied to the length to allow the proportionality to vary with the energy. The following unit length for Coulomb is by definition.

$$
\hat{l}_{e} \equiv k \hat{l}_{g}
$$

Where $\hat{l}_{e}$ is a function of $\hat{l}_{g}$. Equation 16 is now written as:

$$
1=k \frac{E_{e}}{E_{g}}=\frac{\left(\frac{e^{2}}{4 \pi \epsilon_{0} k \hat{l}_{g}}\right)}{M c^{2}}=\frac{e^{2}}{4 \pi \epsilon k \hat{l}_{g} M c^{2}}
$$

Substituting equation (17) into equation (13) and setting the forces equal:

$$
\vec{F}_{g}=\vec{F}_{e}=\frac{e^{2}}{4 \pi \epsilon_{0} k^{2} \hat{l}_{g}^{2}}
$$

Using equation 19 and substituting $\vec{F}_{g}$ with gravitational force and rearranging

$$
\frac{e^{2}}{4 \pi \epsilon_{0} k^{2} \hat{l}_{g}^{2}}=\frac{G M^{2}}{\hat{l}_{g}{ }^{2}}
$$

Solving 20 for $\mathrm{M}$

$$
M=\sqrt{\frac{e^{2}}{4 \pi \epsilon_{0} k^{2} G}}
$$

From equation 8 , one may derive a value for mass M based on $\hat{l}_{g}$.

$$
M=\frac{\hat{g}_{g} c^{2}}{G}=\sqrt{\frac{e^{2}}{4 \pi \epsilon_{0} k^{2} G}} \stackrel{\text { yields }}{\longrightarrow} k M=\sqrt{\frac{e^{2}}{4 \pi \epsilon_{0} G}}
$$

From equation 19 and equation 10:

$$
\vec{F}_{g}=\frac{c^{4}}{G} \stackrel{\text { yields }}{\longrightarrow} k \hat{l}_{g}=\sqrt{\frac{G e^{2}}{4 \pi \epsilon c^{4}}}
$$

From equation 17, $\hat{l}_{e}$ is $k \hat{l}_{g}$. The ratio of forces (Coulomb force to Newtonian gravitational force) is

$$
k^{2}=\frac{\vec{F}_{e}}{\vec{F}_{g}}(24)
$$

With the derived Coulomb units (denoted by a subscript e), Table 1 is updated to show both sets of units. One based on a mass with no charge, and 
the other with a massless particle with mass derived from the energy equivalence of an elementary charge.

\begin{tabular}{|c|c|c|}
\hline Unit & Gravitation Value & Coulomb Value \\
\hline Mass & $\widehat{M}$ & $M_{e}=k \widehat{M}$ \\
\hline Length & $\hat{l}_{g}=\frac{G M}{c^{2}}$ & $\hat{l}_{e}=k \hat{l}_{g}=\frac{G M_{e}}{c^{2}}$ \\
\hline Time & $\hat{t}_{g}=\frac{G M}{c^{3}}$ & $\hat{t}_{e}=k \hat{t}_{g}=\frac{G M_{e}}{c^{3}}$ \\
\hline Unit Force & $\overrightarrow{F_{g}}=\frac{c^{4}}{G}$ & $\overrightarrow{F_{e}}=k^{-2} \vec{F}_{g}=\frac{e^{2}}{4 \pi \epsilon_{0} \hat{l}_{e}^{2}}$ \\
\hline Unit Energy & $\hat{E}_{g}=M c^{2}$ & $\hat{E}_{e}=k \hat{E}_{g}=M_{e} c^{2}$ \\
\hline $\begin{array}{c}\text { A Unit Energy for a Unit } \\
\text { Time }\end{array}$ & $\hat{E}_{g} \hat{t}_{g}=\frac{G M^{2}}{c}$ & $\hat{E}_{e} \hat{t}_{e}=k^{2} \hat{E}_{g} \hat{t}_{g}=\frac{e^{2}}{4 \pi \epsilon_{0} \hat{l}_{e}}$ \\
\hline
\end{tabular}

Table 2

Working with derived units, angular momentum of a single particle in motion about a central force for gravitational and Coulomb force is (Worthington 1906)

Thus

$$
\begin{aligned}
& L_{g}=r m v=\hat{l}_{g} \widehat{M} c \\
& L_{e}=r m v=\hat{l}_{e} M_{e} c
\end{aligned}
$$

$$
L_{e}=k^{2} L_{g}
$$

Angular momentum $L$ in base units for a single particle in motion about a central force is equivalent to Et, a base unit of energy delivered for a base unit of time.

\section{Quanta of Energy}

Using the derived units, the smallest "quanta" of energy measurable is the unit energy delivered for a unit time. Thus, one may infer L (angular momentum) as a quanta of energy when derived from base units. Considering equation 27, the Coulomb quanta of energy is massless, where the gravitational quanta of energy is based on mass. As such, any mass added to the Coulomb angular momentum will reduce its velocity from c. Here a hypothesis is presented, because the mass will reduce the velocity to less than $\mathrm{c}$ and the unit gravitational angular momentum has mass of velocity c, this paper will hypothesis $\mathrm{k}$ is less than one. form

The quanta of energy is represented by $\mathcal{E}$. Equation 27 now takes the

$$
\varepsilon_{e}=k^{2} \varepsilon_{g}
$$

The quanta of energy in Coulomb units is less than the quanta of energy of of gravitational units (based on the hypothesis $\mathrm{k}$ is less than one). Thus

$$
k^{2}=\frac{\varepsilon_{e}}{\varepsilon_{g}}(29)
$$


The squared value of $\mathrm{k}$ is the ratio of quanta of energy for Coulomb derived units to quanta of energy for gravitational derived units. This ratio is not radial dependent as seen by

$$
k^{2}=\frac{\left(\frac{e^{2}}{4 \pi \epsilon}\right)}{G M^{2}}
$$

Consider the equation below from Table 2

$$
\hat{E}_{e} \hat{t}_{e}=k^{2} \hat{E}_{g} \hat{t}_{g}=\frac{e^{2}}{4 \pi \epsilon_{0} \hat{l}_{e}}(31)
$$

By applying the definition for $\hat{l}_{e}$ from Table 2 and substituting the value for $M_{e}$ from equation $21 ; \hat{E}_{e} \hat{t}_{e}$ has a constant value of

$$
\hat{E}_{e} \hat{t}_{e}=k^{2} \hat{E}_{g} \hat{t}_{g}=\frac{e^{2}}{4 \pi \epsilon_{0} c}=7.696 \cdot 10^{-37}
$$

\section{The Hydrogen Atom}

Bohr assumed the angular momentum of the electron must be a whole number $\mathrm{n}$ of $\hbar$, Planck's reduced constant (Bohr 1913).

$$
L_{\text {electron }}=n \hbar(33)
$$

due to the quantization of light. The same calculation of Bohr's radius may be derived with the assumption there is a quantization of gravitational energy (as previously derived) and a continuation of light. Of interest is the consideration that Bohr's radius is determined by the Coulomb force acting on the electron. The Coulomb unit of angular momentum compared to gravitational angular momentum is (from equation 26 with assumed constants applied)

Solving for $r_{e}$

$$
k r_{e} k m_{e} c=r_{g} m_{g} c \text { (34) }
$$

From equations 25 and 31

$$
r_{e}=\frac{r_{g} m_{g} c}{k^{2} m_{e} c}(35)
$$

$$
\hat{E}_{g} \hat{t}_{g}=r_{g} m_{g} c
$$

Where $\hat{E}_{g} \hat{t}_{g}$ is the quanta of energy. Substitute equation 36 into equation 35

$$
r_{e}=\frac{\hat{E}_{g} \hat{t}_{g}}{k^{2} m_{e} c}(37)
$$

By substituting the mass (of the angular momentum caused by the Coulomb force) with the mass of an electron, $r_{e}$ in equation 35 is the classical orbital radius of an electron about the Hydrogen atom. Considering the equation 37, rearranged:

$$
\hat{E}_{g} \hat{t}_{g}=k^{2} r_{e} m_{e} c
$$

With $r_{e}$ and $m_{e}$ known (from equation 25 or equation 26), velocity is

$$
v_{e}=k^{2} c(39)
$$

Here the first orbital of the Hydrogen atom in a semi-classical manner is derived. 


\section{Measurement}

Observation and measurements consequences of equations 6 and 7 model a level of uncertainty. If an observer of an event has no measurable effects due to gravitational potential (a great distance from the massive object), and the event observed does have a mesurable effect of gravitational potential (near the massive object), the best certainty of measurement is equation 7 . Equation 7 limits the certainty to

$$
l=\left(\frac{l^{\prime}}{2}\right)
$$

This uncertainty occurs as the observed length goes to the gravitational unit length of equation 8. A minimum change in distance (unit length) multiplied by a minimum change in momentum is a constant.

$$
\frac{G M}{c^{2}} \cdot M c=\frac{G M^{2}}{c}(41)
$$

The same constant value is found when a minimum energy change (unit energy) is made over a minimum time change (unit time).

$$
\frac{G M}{c^{3}} \cdot M c^{2}=\frac{G M^{2}}{c}(42)
$$

Equations 41 and 42 represent a minimum level of uncertainty in measurement between observers. This is when each observer is at a limit $(\mathrm{r}=$ $\frac{G M}{c^{2}}$ or $\mathrm{r}=$ infinity)

$$
\text { uncertainty }=\Delta x \Delta p=\Delta E \Delta t=\left(\frac{G M^{2}}{c}\right)
$$

\section{Empirical data}

Empirically there are constants which currently must be measured to obtain a value. $G$, the Newtonian gravitational constant is one example (NIST CODATA 2014). Considering equation 32, value $k^{2}\left(\hat{E}_{g} \hat{t}_{g}\right)$ is the product of two unknown constants $k^{2}$ and $\left(\hat{E}_{g} \hat{t}_{g}\right)$ yielding a constant. Secondly, considering the derivation of the Hydrogen atom and the classically derived radius (Bohr 1913), equations 37, 38, and 39 . Lastly, consider the uncertainty of measurement, equation 43. Two strong candidates for the constants $k^{2}\left(\hat{E}_{g} \hat{t}_{g}\right)$ are the fine structure constant $\alpha$ and Planck's reduced constant $\hbar$ meeting the criteria for all constriants. Consider

$$
\alpha \hbar=\frac{e^{2}}{4 \pi \epsilon_{0} c}(42)
$$

These constants maintain units for equation 32. $\alpha$ is a unit-less value, $k^{2}$ is a unit-less value. $\hbar$ has energy and time units, $\hat{E}_{g} \hat{t}_{g}$ has energy and time units. $\hbar$ is a quanta when measuring angular frequency, same as $L_{g}=\hat{E}_{g} \hat{t}_{g}$.

The consequences of substituting these two constants into equation 33 is.

$$
k^{2}=\alpha(43)
$$




$$
\begin{gathered}
\hat{E}_{g} \hat{t}_{g}=\hbar \\
\widehat{M}=M_{p} \\
\hat{l}_{g}=l_{p} \\
\hat{t}_{g}=t_{p} \\
\hat{F}_{g}=F_{p} \\
\hat{E}_{g}=E_{p}
\end{gathered}
$$

Where the subscript $\mathrm{p}$ represents Planck's units, including Planck derived units.

Equation 38 now reads

$$
\hbar=\alpha r_{e} m_{e} c(50)
$$

This is the same as Bohr's classical Hydrogen electron orbital radius:

$$
r_{e}=\frac{\hbar}{\alpha m_{e} c}=5.29 \cdot 10^{-11} \text { meters (52) }
$$

With $r_{e}$ and $m_{e}$ known, the orbital velocity in equation 39 is predicted correctly as (Bohr 1913)

$$
v_{e}=\alpha c(52)
$$

Lastly, substituting into equation 43 , the uncertainty of measurement takes the form of

$$
\text { Uncertainty }=\frac{\hbar}{2}(53)
$$

Which equals Heisenberg's uncertainty (Heisenberg 1927).

\section{Summary}

It has been shown under certain conditions the Schwarzschild's metric has a quanta of length between observers. A constant length, independant of radial distance $\left(\frac{G M^{2}}{c}\right)$ is part of any transformation. From this constant, units of length, time, mass, momentum, force, energy and angular momentum were derived. Elementary charge was introduced and a ratio of gravitational units to Coulomb derived units calculated. From this calculation a quanta of energy was considered. Secondly, from the calculation an equation for an electron's orbit semi-classically derived values were obtained for the Hydrogen atom. Lastly, an uncertainty in measurement was derived as a result of the derived units and the transformation.

These equations contained two unknown variables. By applying empirical data, a hypothesis was set forth, that their values are Planck's constant and the fine structure constant. Upon substitution of the values, all equations matched known data and derived Planck's units. These can be derived from two previous papers (Austin 2017, Austin2, 2017), but it does not use Schawarzschild's solution, thus, they were not used. 
This walk through shows a plausable method by which to derive a quanta of energy, Planck's units, Heisenberg's uncertainty and Bohr's semiclassical orbital radius of an electron about a proton in an Hydrogen atom from Schwarzschild's solution to Einstein's field equations. Further investigation is recommended.

\section{References:}

1. Schwarzschild (1916) Uber das Gravitationsfeld eines Massenpunktes nach der Einsteinschen Theorie Sitzungsberichte der Koniglich Preussischen Akademie Der Wissenschaften

2. NIST CODATA (2014) National Institute of Standards and Technology

3. Marion; Thornton (1995) Classical Dynamics of particles and systems ( $4^{\text {th }}$ ed.) Harcourt Brace \& Company

4. Halliday, David; Resnick, Robert; Walker, Jearl (1997) Electric Potential Fundamentals of Physics ( $5^{\text {th }}$ Edition) John Wiley \& Sons

5. Worthington, Arthur (1906) Dynamics of Rotation, Longmans, Green and Co. London

6. Bohr, Niels (1913) On the Constitution of Atoms and Molecules, Part 1. Philosophical Magazine

7. Heisenberg, W. (1927) Uber den anschaulichen Inhalt der quanterntheoretischen Kinematik und Mechanik, Zeitschrift Fur Physik

8. Austin, Rickey (2017 - June) Metric for a Temporal Manifold Derived from Special Relativity and Newtonian Classical Gravitational Potential. European Science Journal

9. Austin, Rickey (2017 - January) Gravitational Time Dilation Derived from Special Relativity and Newtonian Gravitational Potential. European Science Journal 